\title{
Effects of polysaccharide-based coatings on postharvest storage life of grape: measuring the changes in nutritional, antioxidant and phenolic compounds
}

\author{
Sara Eshghi ${ }^{1} \cdot$ Rouhollah Karimi $^{2}$ (1) $\cdot$ Azam Shiri $^{1} \cdot$ Mostafa Karami $^{1,3} \cdot$ Mohammad Moradi $^{1}$
}

Received: 21 November 2021 / Accepted: 22 December 2021 / Published online: 3 January 2022

(c) The Author(s), under exclusive licence to Springer Science+Business Media, LLC, part of Springer Nature 2022

\begin{abstract}
In this study, the effect of postharvest coating of chitosan $(\mathrm{CH}) 1.0 \%$, gum ghatti (GG) $1.0 \%$ and combine of each other, on nutritional properties, phenolic compounds and antioxidant capacity of 'Rishbaba' grape (Vitis vinifera L.) was evaluated during 60 days of cold storage. Coating with $1.0 \% \mathrm{CH}$ solely or in combined with $1.0 \%$ GG caused a considerable retain in grape berries phenolic acids compared to uncounted samples after the 60th day. Moreover, flavanols and flavan-3-ols content were found to be highest in fruits treated with $\mathrm{CH}$ and GG complex. At the end of storage, the highest concentrations of delphinidin, cyanidin, pelargonidin and malvidin were found in grapes coated with $\mathrm{CH}$ in combined with GG. The highest antioxidant capacity and the lowest polyphenol oxidase activity were related to samples treated with $\mathrm{CH}$ and GG complex. Also, the combination effects of $\mathrm{CH}$ and GG at $1.0 \%$ were the most efficient for soluble sugars and polyamines accumulation. The $\mathrm{CH}+\mathrm{GG}$ complex had the best result on prohibiting grape fungal decay. The results showed a research increase of this complex that these are a strong potential strategy to produce coatings for improving the postharvest quality of fruits and could be considered as a good solution to preserve many components of them.
\end{abstract}

Keywords Grape $\cdot$ Flavanols $\cdot$ Gum ghatti $\cdot$ Phenolic acids $\cdot$ Polyamines $\cdot$ Soluble sugars

\section{Introduction}

Table grapes are non-climacteric fruits with a relatively low physiological activity that widely cultivated in the world. 'Rishbaba' cultivar is one of the high-quality seeded table grapes cultivars produced in Iran. This kind of grape is sweet and juicy, has a good potential for postharvest storage due to its thick skin and fleshy berry [1]. Rishbaba grapes are considered as major sources of phenolic compounds (as bioactive nutrients), minerals, soluble sugars and polyamines

Rouhollah Karimi

R.Karimi@malayeru.ac.ir; Rouholahkarimi@gmail.com

1 Grape Processing and Preservation Department, Research Institute for Grapes and Raisin, Malayer University, Malayer, Iran

2 Grape Production and Genetic Improvement Department, Research Institute for Grapes and Raisin, Malayer University, Malayer, Iran

3 Department of Food Science and Technology, Faculty of Food Science and Technology, Bu-Ali Sina University, Hamedan, Iran that possess oxygen radical scavenging and protective agents against cardiovascular, degenerative diseases and cancer. These compounds are quantified in some fruits and show a high correlation with antioxidant activity [2].

Increased use of grapes in the world has encouraged further investigation into the development of effective postharvest conditions to preserve these fruits. Unfortunately, grape berries are easily dehydrated and their losses are high during transportation and shelf, which is due to high susceptibility to enzymes activity (such as polyphenol oxidase) and pathogenic infection [3]. Botrytis cinerea causes gray mold rot and it is the main cause of postharvest disease in grape fruit. It is possible to control it efficiently using synthetic chemical fungicides (for example $\mathrm{SO}_{2}$ and polyamines), physical (such as UV-C and $\mathrm{Co}_{2}$ ) and the biological preservative (Aureobasidium pullulans and Hanseniaspora uvarum) [4]. However, due to the negative effects of fungicides residues in the environment on human health, there is a great demand for new measures to control postharvest diseases of grape [3]. Fungicides spray such as $\mathrm{SO}_{2}$, is the most commonly used, delaying decay of grapes. However, due to the health 
hazards of using fungicides, their use is restricted and there is a need for natural substitutes [5].

One of the natural techniques to preserve fruits is to use edible coatings, groups of ecofriendly biodegradable compounds based on organic materials such as polysaccharides (chitosan, pectin, starch), other biopolymers featured and some of the gums (such as guar gum, gum ghatti, quince seed gum and etc.). They have the low toxicity to mammalian cells, antimicrobial activity and preservation of bioactive ingredients [6-8]. Edible coatings are considered as the liquid form of edible materials, which can also have other biochemical particles such as nanoparticles. In recent years, successful application of nanotechnology in a wide range of science had been the main incentive to employ this technology in production of packaging materials with a variety of purposes. Mixtures of nanoparticles with packaging materials imply antimicrobial effects that may enhancement of the shelf life of some products [9].

The post-harvest quality and extend the shelf life of 'Rishbaba' grapes can be improved by edible coatings that are good options. Researches have shown that some coatings have potentially positive effects on metabolic changes of fruit such as enhancements of functional substances and efficiently improve the quality of horticultural commodities $[4,10]$. Chitosan $(\mathrm{CH})$, as a cationic polysaccharide, is a natural polymer, which is commercially available through deacetylation of chitin from the exoskeleton of crustaceans. The $\mathrm{CH}$ coatings prove a good potential to lower fungal rot and reduce gas exchanges and aroma. In addition, it is a good option as an internal quality preservative and may demonstrate to be a promising green material owing to its renewability [11].

Recently, Gum ghatti (GG) or Indian gum, is an exudate gum from the tree Anogeissus Indifolia, has received attention. GG has desirable oil and water emulsification stability. GG can be new material in the preparation of edible coatings and films. Joshi et al. showed that by addition of clove oil to an edible coating emulsion based on GG, the shelf life of papaya was extended following by maintaining its quality [12]. The addition of GG to $\mathrm{CH}$ the coating can improve bioactive nutrients of grapes and prolong antimicrobial activity, also the effects of $\mathrm{CH}$ and GG coating as a less hazardous method, have not been reported in previous researches.

The novelties of the present study that less attended in previous researches are including develop new edible coatings based on $\mathrm{CH}$ and/or GG, determine their effect on individual phenolic acid, flavonols, flavan-3-ols, anthocyanin, soluble sugars, polyamines, antioxidant capacity, polyphenol oxidase activity and fungal decay of 'Rishbaba' grapes during cold storage. In addition, these coatings prevent contamination of grapes to avoid the economic losses.

\section{Materials and methods}

\section{Fruit materials and treatments}

Table grape fruits (Vitis vinifera L. Rishbaba) were provided from a local market in Malayer, Iran. The fruits with no sign of mechanical damage and decay were chosen and standardized in small bunches of similar color, size and form. The CH and GG (with Food Grade) were obtained from Sigma Aldrich (Germany). The experiments were conducted in three replications. Treatments contained $1.0 \% \mathrm{CH}$ and $1.0 \%$ GG at four storage times (0, 20, 40 and 60 days). The different $\mathrm{CH}$ and $\mathrm{GG}$ coating treatments were named $\mathrm{CK}$ (GG $0 \%+\mathrm{CH} 0 \%$; control-distilled water); GG (GG 1.0\% + $\mathrm{CH}$ $0 \%) ; \mathrm{CH}(\mathrm{CH} 1.0 \%+\mathrm{GG} \mathrm{0 \%}) ; \mathrm{CH}+\mathrm{GG}(\mathrm{GG} 1.0 \%+\mathrm{CH}$ $1.0 \%)$. Based on the results of a previous study by abovementioned researchers, coating with a concentration of $1.0 \%$ $\mathrm{CH}$ and $1.0 \%$ GG was more efficient on biophysical and safety properties of grape postharvest storage, so we used this concentration [13].

\section{Formulations of edible coatings}

Chitosan solutions were prepared by dissolving $1.0 \%$ of $\mathrm{CH}$ in $2 \mathrm{~mL}$ of glacial acetic acid (1\%) and dissolving by continuous shaking at $55^{\circ} \mathrm{C}$ for $30 \mathrm{~min}$ [14]. Gum ghatti solutions were prepared at $1.0 \% \mathrm{w} / \mathrm{v}$ in distilled water and shook at $55^{\circ} \mathrm{C}$ for $30 \mathrm{~min}$. Afterward, $\mathrm{CH}$ and GG solutions were mixed with each other to prepare planed combination treatments after stirring for $30 \mathrm{~min}$ [13].

\section{Application of coating}

The grape fruits were randomly divided into four groups (control and treatments). The fruits were dipped in the $\mathrm{CH}$, GG or $\mathrm{CH}+\mathrm{GG}$ solution for $5 \mathrm{~min}$ and then were air-dried at $20{ }^{\circ} \mathrm{C}$ for one hour. Finally, approximately 800 gr grape bunches packed in polyethylene plastic containers (dimensions $21 \mathrm{~cm} \times 14 \mathrm{~cm} \times 8 \mathrm{~cm}$ ). The packed grapes were stored in the refrigerator under temperature conditions of 1 $\pm 0.5^{\circ} \mathrm{C}$ and relative humidity of $85-90 \%$ for 60 days. Every 20 days (storage times were $0,20,40$ and 60 days), approximately $200 \mathrm{gr}$ of samples were taken randomly from packed samples in the refrigerator for the following quality analysis.

\section{Phenolic compounds, total phenol and flavonoid}

In this study, phenolic compounds including phenolic acids, resveratrol and flavonoids were determined based on the method explained by Koponen et al. [15] with some changes. For analysis of phenolic acids, $3 \mathrm{~g}$ of each treatment were 
boiled in 0.1 N HCL for 25-30 min. Absorbance was measured at $254 \mathrm{~nm}$. Potassium dihydrogen phosphate and acetonitrile $(80: 20, \mathrm{v} / \mathrm{v})$ were in mobile phase. The flow rate was

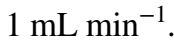

To extract total phenolic and flavonoid contents $10 \mathrm{~g}$ of fruit samples, homogenized in $10-15 \mathrm{~mL}$ of methanol (80\%) and sodium bisulfate $(0.5 \%)$ and centrifuged $(12,000 \mathrm{rpm}$, $15 \mathrm{~min}$ ) at ambient temperature, then filtered and repeated. Total phenolic contents were determined following Singleton and Rossi, with a few changes [16]. The level of absorbance was determined at $765 \mathrm{~nm}$. Based on gallic acid equivalents (GAE) standard curve the total phenolic contents were calculated and expressed as $\mathrm{mg} \mathrm{kg}^{-1}$ of fresh weight. Flavonoid contents were measured following Zhishen et al. so that, the absorbance was measured at $415 \mathrm{~nm}$ with a spectrophotometer (Varian Cary 300, USA) [17]. A standard curve of quercetin was used for calculating this parameter, which was expressed in $\mathrm{mg} \mathrm{kg}^{-1}$.

\section{Anthocyanidins and total anthocyanins}

Extraction procedure of anthocyanidins was performed following Downey et al. using $5 \mathrm{~g}$ of the skins, with $10 \mathrm{~mL}$ of the solvent including $1 \%(\mathrm{w} / \mathrm{v}) \mathrm{HCl}$ in methanol and water mixture (60:40), and then it was left for $10 \mathrm{~min}$ [18]. Anthocyanidins (delphinidin-3-glucoside, cyanidin-3-glucoside, pelargonidin-3 glucoside, malvidin-3-glucoside) were determined using a HPLC system. Spectra were recorded at $518 \mathrm{~nm}$.

Total anthocyanin concentration was measured following Krizek et al. [19] and modified as required. Then, $10 \mathrm{~mL}$ of methanol containing $\mathrm{HCl}(1 \%(\mathrm{v} / \mathrm{v})$ was mixed with $0.1 \mathrm{~g}$ of the grape; after that, it was homogenized and centrifuged at $12,000 \mathrm{rpm}$ for $15 \mathrm{~min}$. After $24 \mathrm{~h}$ in the dark place, measurements were done at $550 \mathrm{~nm}$ with a spectrophotometer (Varian Cary 300, U.S.A). The extinction coefficient for anthocyanin was $33,000 \mathrm{~mol}^{-1} \mathrm{~cm}^{-1}$.

\section{Antioxidant capacity}

The DPPH radical scavenging capacity was measured using 2, 2-diphenyl-1-picrylhydrazyl (DPPH). In brief, $10 \mu \mathrm{L}$ of grape extract was mixed with $3 \mathrm{~mL}$ of $0.1 \mathrm{mM}$ DPPH solution. The mixture was shaken and then left in the dark for $1 \mathrm{~h}$. The decline in absorbance was determined at $517 \mathrm{~nm}$ (using a UV-visible spectrophotometer, Varian Cary 300, U.S.A) [20]. The antioxidant capacity was determined as follows:

$\operatorname{DPPH}(\%)=[($ Ablank - Asample $) /$ Ablank $] \times 100$.

\section{Polyphenol oxidase}

The activity of polyphenol oxidase (PPO) was measured spectrophotometrically at $420 \mathrm{~nm}$ for $10 \mathrm{~min}$ (Spekol 2000, Analytic Jena, Germany) in all samples [21].

\section{Polyamines}

For determination of polyamines, $2 \mathrm{~g}$ of frozen sample was homogenized in $2 \mathrm{~mL}$ of $4 \% \mathrm{HClO}_{4}$ including 1,7 diaminoheptane- $2 \mathrm{HCl}$ as standard. Following $1 \mathrm{~h}$ at $4{ }^{\circ} \mathrm{C}$, the samples were filtrated and then $1 \mathrm{~mL}$ of carbonate buffer $(\mathrm{pH} 9)$ and $1 \mathrm{~mL}$ of dansyl chloride solution were added to $0.2 \mathrm{~mL}$ of the mixture. When one hour at $60{ }^{\circ} \mathrm{C}$ passed, the dansylated polyamines (putrescine, spermidine and spermine) were extracted. Acetonitrile/ $\mathrm{H}_{2} \mathrm{O}(72 / 28, \mathrm{v} / \mathrm{v})$ were in mobile

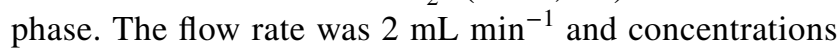
were expressed in $\mathrm{nmol} \mathrm{g}^{-1}$ fresh weight (FW) [22].

\section{Soluble sugars}

Homogenized $1.5 \mathrm{~g}$ of samples with $10 \mathrm{~mL} 80 \%$ ethanol was centrifuged at $8000 \mathrm{rpm}$ for $15 \mathrm{~min}$ and filtered to measure fructose, glucose, and sucrose. Concentrations of fructose, glucose, and sucrose were expressed in $\mu \mathrm{mol} \mathrm{g}^{-1}$ fresh weight [23].

\section{Nutrient content}

Grapes were dried in $72{ }^{\circ} \mathrm{C}$ for $48 \mathrm{~h}$ and used for evaluation of potassium $(\mathrm{K})$, magnesium $(\mathrm{Mg})$, calcium $(\mathrm{Ca})$, zinc $(\mathrm{Zn})$, manganese $(\mathrm{Mn})$, iron $(\mathrm{Fe})$. Potassium was measured by flame photometer (SKZ1044A K Na Digital Flame Photometer., China) and other minerals were measured through atomic absorption spectrophotometer (contrAA 700, Russia, Kurkino).

\section{Microbial analysis}

In this study, molds and yeasts were determined based on the method explained by Jafari et al. [24] with some changes. To evaluate the molds and yeasts in the grapes, $10 \mathrm{~g}$ of each sample was mixed in $90 \mathrm{ml}$ of sterile physiological serum and homogenized. Then, $0.1 \mathrm{~mL}$ sample of serial dilutions of grape homogenates were spread on plates of PDA. The Petri-dishes were incubated at $25{ }^{\circ} \mathrm{C}$ for 5 days and yeast and mold counts of grapes were expressed as log colony forming unit) $\mathrm{CFU}\left(\mathrm{g}^{-1}\right.$.

\section{Statistical analysis}

The collected data was analyzed using ANOVA in SAS software (SAS Institute Inc., Cary, NC, USA) and differences 
among means were determined by the Tukey's multiple range test using a significance level of 0.05 .

\section{Results}

\section{Phenolic acids and total phenolic}

The phenolic acids in grapes are the main compounds for health benefits. The phenolic acids and total phenolic of the berries treated with $\mathrm{CH}$ with and without GG are shown in Table 1. Phenolic acids such as gallic acid, caffeic acid, p-coumaric acid, ferulic acid and resveratrol (as a natural stilbene in grape) are mainly present in grapes. All of the phenolic acids were decreased during storage time (Table 1). Results showed that resveratrol and p-coumaric acid were very important substances extracted from phenolic acids that had the highest levels. The resveratrol and p-coumaric acid of uncoated grape decreased from $11.9,10.1 \mu^{-1} \mathrm{~g} \mathrm{~g}^{-1} \mathrm{FW}$ to 9.4, 8.2 $\mu \mathrm{g} \mathrm{g}^{-1} \mathrm{FW}$ during 60 days but in the coated grapes, these changes in value were less so that in $\mathrm{CH}+\mathrm{GG}$ coated on the $60^{\text {th }}$ day, it is still over $9.5 \mu \mathrm{g} \mathrm{g}^{-1} \mathrm{FW}$. Other phenolic acids such as gallic acid, caffeic acid and ferulic acid, which were first measured in 'Rishbaba' grape, also showed a much lower reduction after 60 days of storage with $\mathrm{CH}+\mathrm{GG}$ coating compared to the $\mathrm{CK}$ sample. For example, caffeic acid decreased from $1.4 \mu \mathrm{g} \mathrm{g}^{-1} \mathrm{FW}$ to $1.2 \mu \mathrm{g} \mathrm{g}^{-1}$ FW during storage, while in the control sample it decreased to less than $1 \mu \mathrm{g} \mathrm{g}^{-1} \mathrm{FW}$. Overall, the grape fruits coated with $\mathrm{CH}$ $+\mathrm{GG}$ and the uncoated sample were significantly different (on average, the amount of phenolic acids in the grape fruits coated with $\mathrm{CH}+\mathrm{GG}$ were almost twice as high as the control sample during 60 days).

The total phenol contents decreased during storage time, which may be due to oxidation of sensitive phenolic compounds. The $\mathrm{CH}+\mathrm{GG}$ treated grapes displayed a significantly different compared to control during cold storage period. The total phenolic content was highest in the grapes coated by $\mathrm{CH}, \mathrm{CH}+\mathrm{GG}$ (more than $1.15 \%$ ) compared to GG coated and control grapes during the $60^{\text {th }}$ day of storage.

\section{Flavonoids and total flavonoid}

In this study, for the first time, the flavonoids in 'Rishbaba' grape were examined separately. All flavonoids were categorized into two groups of flavan-3-ols and flavanols (flavanols such as myricetin, quercetin, kaempferol and flavan-3-ols such as catechin, catechin hydrate, and epicatechin). The flavanols and flavan-3-ols of the berries treated with $\mathrm{CH}$ with and without GG are shown in Table 2. In our research, the flavonoids decreased during storage time. For example, catechin from the flavanols category and quercetin from flavan-3-ols category had highest levels that at the first time of storage both of them were $13 \mu \mathrm{g} \mathrm{g}^{-1} \mathrm{FW}$ and during 60 days they were decreased. On the $60^{\text {th }}$ day, in uncoated grape catechin and quercetin were 4.9 and $10.4 \mu \mathrm{g} \mathrm{g}^{-1} \mathrm{FW}$ but in

Table 1 The effect of pretreatment with chitosan and gum ghatti on phenolic acids, resveratrol and total phenolic of 'Rishbaba' grape stored at 0 $\pm 1^{\circ} \mathrm{C}$

\begin{tabular}{|c|c|c|c|c|c|c|c|}
\hline Treatments & Day & $\begin{array}{l}\text { Gallic acid ( } \mu \mathrm{g} \\
\left.\mathrm{g}^{-1} \mathrm{FW}\right)\end{array}$ & $\begin{array}{l}\text { Caffeic acid }(\mu \mathrm{g} \\
\left.\mathrm{g}^{-1} \mathrm{FW}\right)\end{array}$ & $\begin{array}{l}\text { p-Coumaric acid } \\
\left(\mu \mathrm{g} \mathrm{g}^{-1} \mathrm{FW}\right)\end{array}$ & $\begin{array}{l}\text { Ferulic acid ( } \mu \mathrm{g} \\
\left.\mathrm{g}^{-1} \mathrm{FW}\right)\end{array}$ & Resveratrol $\left(\mu \mathrm{g} \mathrm{g}^{-1} \mathrm{FW}\right)$ & $\begin{array}{l}\text { Total phenolic } \\
\left(\mathrm{mg} \mathrm{g}^{-1} \mathrm{FW}\right)\end{array}$ \\
\hline $\mathrm{CK}$ & 0 & $5.9 \pm 0.07 \mathrm{a}$ & $1.4 \pm 0.02 \mathrm{a}$ & $10.1 \pm 0.03 a$ & $3.9 \pm 0.04 \mathrm{a}$ & $11.9 \pm 0.03 \mathrm{a}$ & $2.5 \pm 0.06 \mathrm{a}$ \\
\hline GG & & $5.9 \pm 0.07 \mathrm{a}$ & $1.4 \pm 0.02 \mathrm{a}$ & $10.1 \pm 0.03 a$ & $3.9 \pm 0.04 \mathrm{a}$ & $11.9 \pm 0.03 \mathrm{a}$ & $2.5 \pm 0.06 \mathrm{a}$ \\
\hline $\mathrm{CH}$ & & $5.9 \pm 0.07 \mathrm{a}$ & $1.4 \pm 0.02 \mathrm{a}$ & $10.1 \pm 0.03 \mathrm{a}$ & $3.9 \pm 0.04 \mathrm{a}$ & $11.9 \pm 0.03 a$ & $2.5 \pm 0.06 \mathrm{a}$ \\
\hline $\mathrm{CH}+\mathrm{GG}$ & & $5.9 \pm 0.07 \mathrm{a}$ & $1.4 \pm 0.02 \mathrm{a}$ & $10.1 \pm 0.03 \mathrm{a}$ & $3.9 \pm 0.04 \mathrm{a}$ & $11.9 \pm 0.03 \mathrm{a}$ & $2.5 \pm 0.06 \mathrm{a}$ \\
\hline CK & 20 & $4.3 \pm 0.04 \mathrm{e}$ & $1 \pm 0.01 \mathrm{e}$ & $9.3 \pm 0.05 \mathrm{~g}$ & $3.8 \pm 0.05 b$ & $10.6 \pm 0.02 \mathrm{~d}$ & $1.5 \pm 0.02 \mathrm{e}$ \\
\hline GG & & $4.5 \pm 0.08 \mathrm{~d}$ & $1.1 \pm 0.01 \mathrm{~d}$ & $9.4 \pm 0.01 \mathrm{f}$ & $3.8 \pm 0.02 b$ & $10.8 \pm 0.07 d$ & $1.59 \pm 0.02 \mathrm{e}$ \\
\hline $\mathrm{CH}$ & & $5.1 \pm 0.07 \mathrm{c}$ & $1.3 \pm 0.03 b$ & $9.8 \pm 0.05 \mathrm{c}$ & $3.9 \pm 0.02 \mathrm{a}$ & $11.6 \pm 0.06 b$ & $2.05 \pm 0.02 \mathrm{c}$ \\
\hline $\mathrm{CH}+\mathrm{GG}$ & & $5.7 \pm 0.1 b$ & $1.3 \pm 0.02 b$ & $9.9 \pm 0.02 b$ & $3.9 \pm 0.02 \mathrm{a}$ & $11.7 \pm 0.04 b$ & $2.15 \pm 0.02 b$ \\
\hline CK & 40 & $4 \pm 0.09 f$ & $1 \pm 0.03 \mathrm{e}$ & $8.5 \pm 0.04 \mathrm{k}$ & $3.4 \pm 0.03 \mathrm{~d}$ & $10.1 \pm 0.05 \mathrm{~g}$ & $1.01 \pm 0.01 \mathrm{~h}$ \\
\hline GG & & $4.2 \pm 0.07 \mathrm{e}$ & $1 \pm 0.02 \mathrm{e}$ & $8.7 \pm 0.01 i$ & $3.4 \pm 0.04 d$ & $10.3 \pm 0.03 f$ & $1.13 \pm 0.02 \mathrm{~g}$ \\
\hline $\mathrm{CH}$ & & $5 \pm 0.03 c$ & $1.2 \pm 0.05 \mathrm{c}$ & $9.2 \pm 0.06 \mathrm{~g}$ & $3.8 \pm 0.01 b$ & $10.6 \pm 0.02 d$ & $1.53 \pm 0.05 \mathrm{e}$ \\
\hline $\mathrm{CH}+\mathrm{GG}$ & & $5.3 \pm 0.04 c$ & $1.3 \pm 0.02 b$ & $9.7 \pm 0.01 \mathrm{~d}$ & $3.8 \pm 0.01 b$ & $11 \pm 0.01 \mathrm{c}$ & $1.73 \pm 0.07 \mathrm{~d}$ \\
\hline CK & 60 & $3.8 \pm 0.12 \mathrm{f}$ & $0.8 \pm 0.01 \mathrm{~g}$ & $8.2 \pm 0.05 \mathrm{k}$ & $3 \pm 0.06 \mathrm{e}$ & $9.4 \pm 0.01 \mathrm{i}$ & $0.85 \pm 0.01 \mathrm{j}$ \\
\hline GG & & $3.9 \pm 0.03 f$ & $0.9 \pm 0.05 f$ & $8.4 \pm 0.02 j$ & $3 \pm 0.05 \mathrm{e}$ & $9.6 \pm 0.06 \mathrm{~h}$ & $0.94 \pm 0.03 \mathrm{i}$ \\
\hline $\mathrm{CH}$ & & $4.6 \pm 0.05 d$ & $1.1 \pm 0.05 \mathrm{~d}$ & $8.9 \pm 0.09 h$ & $3.3 \pm 0.05 d$ & $10.1 \pm 0.04 \mathrm{~g}$ & $1.15 \pm 0.03 \mathrm{~g}$ \\
\hline $\mathrm{CH}+\mathrm{GG}$ & & $5 \pm 0.1 \mathrm{c}$ & $1.2 \pm 0.01 \mathrm{c}$ & $9.5 \pm 0.04 \mathrm{e}$ & $3.5 \pm 0.03 c$ & $10.5 \pm 0.05 \mathrm{e}$ & $1.29 \pm 0.03 \mathrm{f}$ \\
\hline
\end{tabular}

The means showing same letters in each column are not different statistically $(\mathrm{P} \leq 0.05)$

Chitosan 0\%-Gum ghatti 0\% (CK); Chitosan 0\%-Gum ghatti 1\% (GG); Chitosan 1\%-Gum ghatti 0\% (CH); Chitosan 1\%-Gum ghatti 1\% (CH + GG) 
Table 2 The effect of pretreatment with chitosan and gum ghatti on flavonoids (flavanols and flavan-3-ols) and total flavonoid of 'Rishbaba' grape stored at $0 \pm 1{ }^{\circ} \mathrm{C}$

\begin{tabular}{|c|c|c|c|c|c|c|c|c|}
\hline \multirow[t]{2}{*}{ Treatments } & \multirow[t]{2}{*}{ Day } & \multicolumn{3}{|c|}{ Flavan-3-ols $\left(\mu \mathrm{g} \mathrm{g}^{-1} \mathrm{FW}\right)$} & \multicolumn{3}{|c|}{ Flavanols $\left(\mu \mathrm{g} \mathrm{g}^{-1} \mathrm{FW}\right)$} & \multirow{2}{*}{$\begin{array}{l}\text { Total flavonoid } \\
\left(\mathrm{mg} \mathrm{g}^{-1} \mathrm{FW}\right)\end{array}$} \\
\hline & & Catechin & Catechin hydrate & Epicatechin & Myricetin & Quercetin & Kaempferol & \\
\hline $\mathrm{CK}$ & 0 & $13 \pm 0.1 \mathrm{a}$ & $0.8 \pm 0.02 \mathrm{a}$ & $1.4 \pm 0.04 \mathrm{a}$ & $1.8 \pm 0.02 \mathrm{a}$ & $13 \pm 0.07 \mathrm{a}$ & $1.4 \pm 0.01 \mathrm{a}$ & $2.01 \pm 0.04 \mathrm{a}$ \\
\hline GG & & $13 \pm 0.1 \mathrm{a}$ & $0.8 \pm 0.02 \mathrm{a}$ & $1.4 \pm 0.04 \mathrm{a}$ & $1.8 \pm 0.02 \mathrm{a}$ & $13 \pm 0.07 \mathrm{a}$ & $1.4 \pm 0.01 \mathrm{a}$ & $2.01 \pm 0.04 \mathrm{a}$ \\
\hline $\mathrm{CH}$ & & $13 \pm 0.1 \mathrm{a}$ & $0.8 \pm 0.02 \mathrm{a}$ & $1.4 \pm 0.04 \mathrm{a}$ & $1.8 \pm 0.02 \mathrm{a}$ & $13 \pm 0.07 \mathrm{a}$ & $1.4 \pm 0.01 \mathrm{a}$ & $2.01 \pm 0.04 \mathrm{a}$ \\
\hline $\mathrm{CH}+\mathrm{GG}$ & & $13 \pm 0.1 \mathrm{a}$ & $0.8 \pm 0.02 \mathrm{a}$ & $1.4 \pm 0.04 \mathrm{a}$ & $1.8 \pm 0.02 \mathrm{a}$ & $13 \pm 0.07 \mathrm{a}$ & $1.4 \pm 0.01 \mathrm{a}$ & $2.01 \pm 0.04 \mathrm{a}$ \\
\hline $\mathrm{CK}$ & 20 & $6.6 \pm 0.09 h$ & $0.7 \pm 0.01 b$ & $1.2 \pm 0.05 \mathrm{c}$ & $1.5 \pm 0.04 \mathrm{~d}$ & $12.1 \pm 0.02 \mathrm{f}$ & $1.1 \pm 0.06 \mathrm{~d}$ & $1.4 \pm 0.03 \mathrm{e}$ \\
\hline GG & & $6.9 \pm 0.08 \mathrm{~g}$ & $0.7 \pm 0.01 b$ & $1.2 \pm 0.02 \mathrm{c}$ & $1.5 \pm 0.02 \mathrm{~d}$ & $12.2 \pm 0.02 \mathrm{e}$ & $1.2 \pm 0.02 \mathrm{c}$ & $1.48 \pm 0.01 \mathrm{~d}$ \\
\hline $\mathrm{CH}$ & & $8.4 \pm 0.12 \mathrm{e}$ & $0.7 \pm 0.03 b$ & $1.3 \pm 0.04 b$ & $1.7 \pm 0.01 b$ & $12.6 \pm 0.03 d$ & $1.4 \pm 0.02 \mathrm{a}$ & $1.68 \pm 0.02 c$ \\
\hline $\mathrm{CH}+\mathrm{GG}$ & & $12.3 \pm 0.06 b$ & $0.8 \pm 0.01 \mathrm{a}$ & $1.4 \pm 0.02 \mathrm{a}$ & $1.8 \pm 0.03 \mathrm{a}$ & $12.8 \pm 0.03 b$ & $1.4 \pm 0.03 a$ & $1.81 \pm 0.02 b$ \\
\hline $\mathrm{CK}$ & 40 & $5.8 \pm 0.1 \mathrm{j}$ & $0.6 \pm 0.03 c$ & $1 \pm 0.03 \mathrm{e}$ & $1.3 \pm 0.03 \mathrm{e}$ & $11.3 \pm 0.03 \mathrm{i}$ & $1 \pm 0.03 d$ & $1.02 \pm 0.01 \mathrm{~g}$ \\
\hline GG & & $6.3 \pm 0.1 \mathrm{i}$ & $0.6 \pm 0.02 c$ & $1 \pm 0.05 \mathrm{e}$ & $1.4 \pm 0.03 \mathrm{e}$ & $11.4 \pm 0.06 h$ & $1.1 \pm 0.03 \mathrm{~d}$ & $1.1 \pm 0.01 \mathrm{f}$ \\
\hline $\mathrm{CH}$ & & $7.7 \pm 0.05 f$ & $0.7 \pm 0.01 b$ & $1.1 \pm 0.01 \mathrm{~d}$ & $1.5 \pm 0.04 d$ & $12.2 \pm 0.05 \mathrm{e}$ & $1.2 \pm 0.01 \mathrm{c}$ & $1.42 \pm 0.03 \mathrm{e}$ \\
\hline $\mathrm{CH}+\mathrm{GG}$ & & $11.8 \pm 0.03 c$ & $0.7 \pm 0.03 b$ & $1.3 \pm 0.01 b$ & $1.6 \pm 0.04 c$ & $12.7 \pm 0.05 c$ & $1.3 \pm 0.02 b$ & $1.65 \pm 0.02 c$ \\
\hline $\mathrm{CK}$ & 60 & $4.9 \pm 0.051$ & $0.4 \pm 0.01 \mathrm{e}$ & $0.7 \pm 0.01 \mathrm{f}$ & $1 \pm 0.05 \mathrm{~g}$ & $10.4 \pm 0.01 \mathrm{k}$ & $0.5 \pm 0.01 \mathrm{f}$ & $0.8 \pm 0.02 \mathrm{i}$ \\
\hline GG & & $5.2 \pm 0.07 \mathrm{k}$ & $0.5 \pm 0.04 d$ & $0.9 \pm 0.03 \mathrm{e}$ & $1.1 \pm 0.03 f$ & $10.5 \pm 0.09 \mathrm{k}$ & $0.7 \pm 0.02 \mathrm{e}$ & $0.87 \pm 0.01 \mathrm{~h}$ \\
\hline $\mathrm{CH}$ & & $6.8 \pm 0.06 \mathrm{~g}$ & $0.6 \pm 0.02 c$ & $1 \pm 0.07 \mathrm{e}$ & $1.3 \pm 0.07 \mathrm{e}$ & $11.2 \pm 0.02 \mathrm{j}$ & $1 \pm 0.05 \mathrm{~d}$ & $1.03 \pm 0.01 \mathrm{~g}$ \\
\hline $\mathrm{CH}+\mathrm{GG}$ & & $10.5 \pm 0.05 d$ & $0.6 \pm 0.04 c$ & $1.2 \pm 0.05 \mathrm{c}$ & $1.5 \pm 0.01 d$ & $11.9 \pm 0.01 \mathrm{~g}$ & $1.2 \pm 0.03 c$ & $1.12 \pm 0.02 f$ \\
\hline
\end{tabular}

The means showing same letters in each column are not different statistically $(\mathrm{P} \leq 0.05)$

Chitosan 0\%-Gum ghatti 0\% (CK); Chitosan 0\%-Gum ghatti 1\% (GG); Chitosan 1\%-Gum ghatti 0\% (CH); Chitosan 1\%-Gum ghatti 1\% (CH + GG)

coated samples especially $\mathrm{CH}+\mathrm{GG}$ showed less decrease. In the case of catechin hydrate and kaempferol, which had lowest levels of total flavonoid contents, a significant difference was observed between the control and $\mathrm{CH}+\mathrm{GG}$ samples after 60 days of storage. Therefore, during 60 days of storage, the total flavonoid contents were highest in the grapes coated by $\mathrm{CH}$ and $\mathrm{CH}+\mathrm{GG}$ (more than $1 \mathrm{mg} \mathrm{g}^{-1}$ FW) compared to GG coated.

\section{Anthocyanidins and total anthocyanin content}

Berry anthocyanidins such as delphinidin-3-glucoside, cyanidin-3-glucoside, pelargonidin-3 glucoside, and malvidin3 -glucoside were also evaluated. From the beginning to the end of storage times, all of the anthocyanidins derivatives decreased (Table 3). Among the coated grapes, $\mathrm{CH}$ and $\mathrm{CH}$ + GG had significantly different variables compared to the untreated samples. At the end of storage time, the highest concentrations were found with the formula $\mathrm{CH}+\mathrm{GG}$ for delphinidin, cyanidin, pelargonidin, malvidin equal to 3.55 , $2.18,3.54,1.93 \mathrm{mg} \mathrm{g}^{-1}$ FW of berry. Data analyses demonstrated a significant difference between some coated and uncoated samples during storage. Total anthocyanin content decreased in both coated and uncoated samples. There was a notable change in the content of anthocyanin in the samples coated with $\mathrm{CH}$ and $\mathrm{CH}+\mathrm{GG}$ (from 67.1 at the first time to $21.6,23.8 \mathrm{mg} \mathrm{Kg}^{-1} \mathrm{FW}$ of berry during 60 days, respectively) and CK sample (from 67.1 at the first time to $18.2 \mathrm{mg} \mathrm{Kg}^{-1}$ FW of berry during 60 days) (Table 3 ).

\section{Antioxidant capacity}

In our study, the grape's antioxidant capacity was decreased during 60 days. As shown in Fig. 1, the DPPH radicals are $55.2 \%$ in the beginning and then slowly decrease for all treatments. A significant difference was observed between grapes treated with $\mathrm{CH}+\mathrm{GG}$ coated and control samples. The antioxidant capacity of $\mathrm{CH}+\mathrm{GG}$ coated berries was significantly higher than $\mathrm{CH}$ alone and $\mathrm{GG}$ alone throughout storage. The fruit coated with $\mathrm{CH}+\mathrm{GG}$ shown the highest antioxidant capacity (25.5\% during 60 days of storage) followed by the grapes coated with $\mathrm{CH}$ (19.5\% during 60 days of storage) and then GG coated.

\section{Polyphenol oxidase activity}

The highest PPO activity was observed $(42.8 \mathrm{U} / \mathrm{mg}$ protein) in the first time. Activity of PPO decreased in all samples during the 20 days of storage. The PPO activity was stopped in all treatments during the 20 days of storage followed by a high-rate decline during the 60 days of shelf life (Fig. 2). On the $60^{\text {th }}$ day of storage, the activity in the control 
Table 3 The effect of pretreatment with chitosan and gum ghatti on anthocyanidins and total anthocyanins of 'Rishbaba' grape stored at $0 \pm$ $1{ }^{\circ} \mathrm{C}$

\begin{tabular}{lcrcrrr}
\hline Treatments & Day & $\begin{array}{l}\text { Delphinidin-3-gluco- } \\
\text { side }\left(\mathrm{mg} \mathrm{g}^{-1} \mathrm{FW}\right)\end{array}$ & $\begin{array}{l}\text { Cyanidin-3-glucoside } \\
\left(\mathrm{mg} \mathrm{g}^{-1} \mathrm{FW}\right)\end{array}$ & $\begin{array}{l}\text { Pelargonidin-3 gluco- } \\
\text { side }\left(\mathrm{mg} \mathrm{g}^{-1} \mathrm{FW}\right)\end{array}$ & $\begin{array}{l}\text { Malvinidin-3-gluco- } \\
\text { side }\left(\mathrm{mg} \mathrm{g}^{-1} \mathrm{FW}^{2}\right.\end{array}$ & $\begin{array}{l}\text { Total antho- } \\
\text { cyanins }\left(\mathrm{mg} \mathrm{g}^{-1}\right. \\
\mathrm{FW})\end{array}$ \\
\hline $\mathrm{CK}$ & 0 & $5.04 \pm 0.03 \mathrm{a}$ & $3.34 \pm 0.01 \mathrm{a}$ & $4.91 \pm 0.05 \mathrm{a}$ & $2.17 \pm 0.01 \mathrm{a}$ & $67.1 \pm 0.2 \mathrm{a}$ \\
$\mathrm{GG}$ & & $5.04 \pm 0.03 \mathrm{a}$ & $3.34 \pm 0.01 \mathrm{a}$ & $4.91 \pm 0.05 \mathrm{a}$ & $2.17 \pm 0.01 \mathrm{a}$ & $67.1 \pm 0.2 \mathrm{a}$ \\
$\mathrm{CH}$ & & $5.04 \pm 0.03 \mathrm{a}$ & $3.34 \pm 0.01 \mathrm{a}$ & $4.91 \pm 0.05 \mathrm{a}$ & $2.17 \pm 0.01 \mathrm{a}$ & $67.1 \pm 0.2 \mathrm{a}$ \\
$\mathrm{CH}+\mathrm{GG}$ & & $5.04 \pm 0.03 \mathrm{a}$ & $3.34 \pm 0.01 \mathrm{a}$ & $4.91 \pm 0.05 \mathrm{a}$ & $2.17 \pm 0.01 \mathrm{a}$ & $67.1 \pm 0.2 \mathrm{a}$ \\
$\mathrm{CK}$ & 20 & $3 \pm 0.01 \mathrm{f}$ & $1.85 \pm 0.02 \mathrm{f}$ & $3.42 \pm 0.03 \mathrm{~d}$ & $1.8 \pm 0.04 \mathrm{~d}$ & $58.5 \pm 0.4 \mathrm{e}$ \\
$\mathrm{GG}$ & & $3.24 \pm 0.04 \mathrm{e}$ & $1.97 \pm .0 .02 \mathrm{f}$ & $3.55 \pm 0.02 \mathrm{c}$ & $1.89 \pm 0.02 \mathrm{~d}$ & $59.1 \pm 0.1 \mathrm{~d}$ \\
$\mathrm{CH}$ & & $3.75 \pm 0.02 \mathrm{c}$ & $2.81 \pm 0.04 \mathrm{c}$ & $4.8 \pm 0.02 \mathrm{a}$ & $2.08 \pm 0.01 \mathrm{~b}$ & $60.8 \pm 0.1 \mathrm{c}$ \\
$\mathrm{CH}+\mathrm{GG}$ & & $3.94 \pm 0.01 \mathrm{~b}$ & $2.99 \pm 0.02 \mathrm{~b}$ & $4.89 \pm 0.02 \mathrm{a}$ & $2.13 \pm 0.01 \mathrm{~b}$ & $62.7 \pm 0.1 \mathrm{~b}$ \\
$\mathrm{CK}$ & 40 & $2.6 \pm 0.05 \mathrm{~h}$ & $1.7 \pm 0.06 \mathrm{f}$ & $2.9 \pm 0.06 \mathrm{f}$ & $1.41 \pm 0.02 \mathrm{e}$ & $43 \pm 0.3 \mathrm{i}$ \\
$\mathrm{GG}$ & & $2.69 \pm 0.02 \mathrm{~g}$ & $1.78 \pm 0.02 \mathrm{f}$ & $3.12 \pm 0.05 \mathrm{e}$ & $1.47 \pm 0.01 \mathrm{e}$ & $44.1 \pm 0.2 \mathrm{~h}$ \\
$\mathrm{CH}$ & & $3.5 \pm 0.07 \mathrm{~d}$ & $2.57 \pm 0.02 \mathrm{~d}$ & $4.51 \pm 0.02 \mathrm{~b}$ & $1.95 \pm 0.03 \mathrm{c}$ & $47.2 \pm 0.3 \mathrm{~g}$ \\
$\mathrm{CH}+\mathrm{GG}$ & & $3.76 \pm 0.05 \mathrm{c}$ & $2.79 \pm 0.03 \mathrm{c}$ & $4.78 \pm 0.02 \mathrm{a}$ & $2.1 \pm 0.02 \mathrm{~b}$ & $49.2 \pm 0.1 \mathrm{f}$ \\
$\mathrm{CK}$ & 60 & $2 \pm 0.02 \mathrm{j}$ & $1.35 \pm 0.04 \mathrm{~g}$ & $2.7 \pm 0.01 \mathrm{~h}$ & $1.4 \pm 0.03 \mathrm{e}$ & $18.2 \pm 0.2 \mathrm{~m}$ \\
$\mathrm{GG}$ & & $2.17 \pm 0.01 \mathrm{i}$ & $1.41 \pm 0.05 \mathrm{~g}$ & $2.81 \pm 0.03 \mathrm{~g}$ & $1.44 \pm 0.02 \mathrm{e}$ & $19.1 \pm 0.081$ \\
$\mathrm{CH}$ & & $3 \pm 0.01 \mathrm{f}$ & $1.78 \pm 0.04 \mathrm{f}$ & $3.1 \pm 0.07 \mathrm{e}$ & $1.78 \pm 0.02 \mathrm{~d}$ & $21.6 \pm 0.3 \mathrm{k}$ \\
$\mathrm{CH}+\mathrm{GG}$ & & $3.55 \pm 0.02 \mathrm{~d}$ & $2.18 \pm 0.01 \mathrm{e}$ & $3.54 \pm 0.03 \mathrm{c}$ & $1.93 \pm 0.01 \mathrm{c}$ & $23.8 \pm 0.2 \mathrm{j}$ \\
\hline
\end{tabular}

The means showing same letters in each column are not different statistically $(\mathrm{P} \leq 0.05)$

Chitosan 0\%-Gum ghatti 0\% (CK); Chitosan 0\%-Gum ghatti 1\% (GG); Chitosan 1\%-Gum ghatti 0\% (CH); Chitosan 1\%-Gum ghatti 1\% (CH + GG)

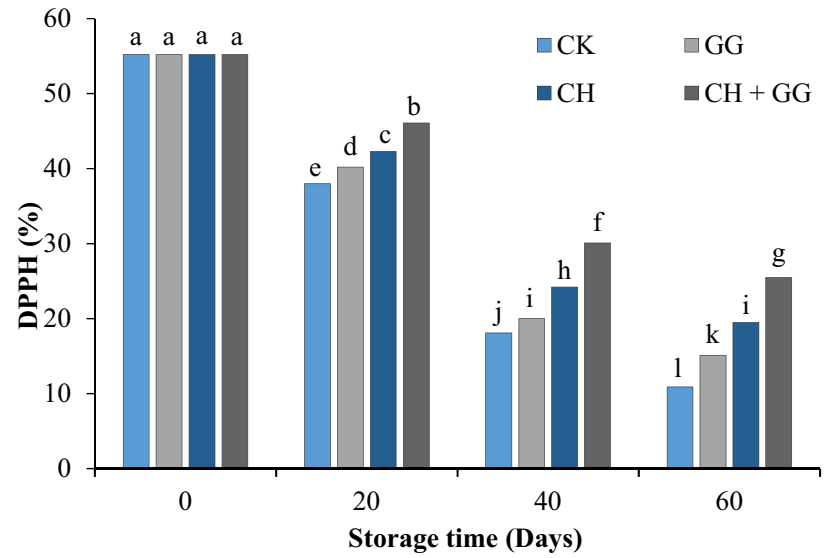

Fig. 1 Effect of chitosan, gum ghatti, chitosan-gum ghatti coatings on antioxidant capacity (assay as DPPH) of Rishbaba grapes stored at $0 \pm 1{ }^{\circ} \mathrm{C}$ with $85 \%$ relative humidity for 60 days. The means showing same letters in each point are not different statistically $(\mathrm{P} \leq 0.05)$. Chitosan 0\%-Gum ghatti 0\% (CK); Chitosan 0\%-Gum ghatti 1\% (GG); Chitosan 1\%-Gum ghatti $0 \%(\mathrm{CH})$; Chitosan $1 \%$-Gum ghatti $1 \%(\mathrm{CH}+\mathrm{GG})$

samples was $6.64 \mathrm{U} / \mathrm{mg}$ protein, this figure was higher in the

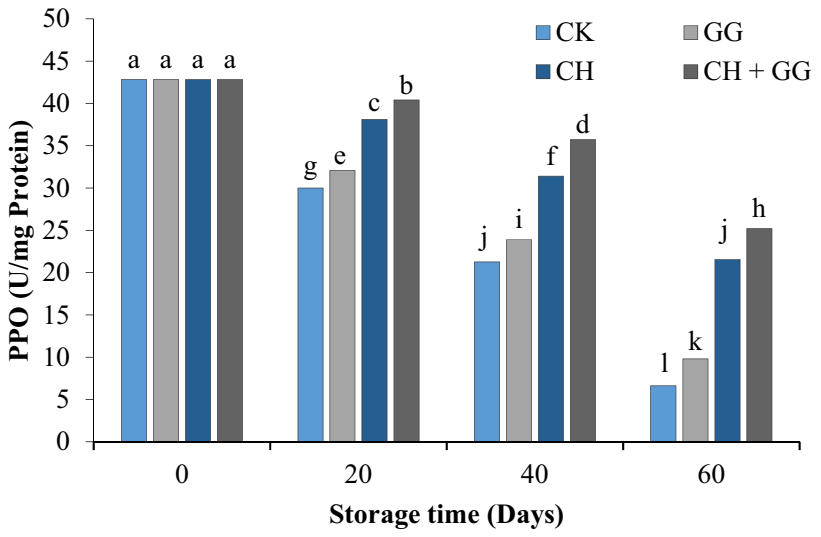

Fig. 2 Effect of chitosan, gum ghatti, chitosan-gum ghatti coatings on polyphenol oxidase activity (PPO) of Rishbaba grapes stored at $0 \pm 1$ ${ }^{\circ} \mathrm{C}$ with $85 \%$ relative humidity for 60 days. The means showing same letters in each point are not different statistically $(\mathrm{P} \leq 0.05)$. Chitosan 0\%-Gum ghatti 0\% (CK); Chitosan 0\%-Gum ghatti 1\% (GG); Chitosan $1 \%$-Gum ghatti $0 \%(\mathrm{CH})$; Chitosan $1 \%$-Gum ghatti $1 \%(\mathrm{CH}+$ GG)

treatment samples. Such observations demonstrate that some coatings in this study (such as $\mathrm{CH}$ and $\mathrm{CH}+\mathrm{GG}$ ) delayed the undesirable changes. 
Table 4 The effect of pretreatment with chitosan and gum ghatti on polyamines of 'Rishbaba' grape stored at $0 \pm$ $1{ }^{\circ} \mathrm{C}$

\begin{tabular}{lcccc}
\hline Treatments & Day & Putrescine $\left(\mathrm{nmol} \mathrm{g}^{-1} \mathrm{FW}\right)$ & \multicolumn{2}{l}{$\begin{array}{l}\text { Spermidine }(\mathrm{nmol} \\
\left.\mathrm{g}^{-1} \mathrm{FW}\right)\end{array}$} \\
\hline $\mathrm{CK}$ & 0 & $12.18 \pm 0.03 \mathrm{a}$ & $21.35 \pm 0.1 \mathrm{a}$ & $20.41 \pm 0.12 \mathrm{a}$ \\
$\mathrm{GG}$ & & $12.18 \pm 0.03 \mathrm{a}$ & $21.35 \pm 0.1 \mathrm{a}$ & $20.41 \pm 0.12 \mathrm{a}$ \\
$\mathrm{CH}$ & $12.18 \pm 0.03 \mathrm{a}$ & $21.35 \pm 0.1 \mathrm{a}$ & $20.41 \pm 0.12 \mathrm{a}$ \\
$\mathrm{CH}+\mathrm{GG}$ & & $12.18 \pm 0.03 \mathrm{a}$ & $21.35 \pm 0.1 \mathrm{a}$ & $20.41 \pm 0.12 \mathrm{a}$ \\
$\mathrm{CK}$ & 20 & $10.01 \pm 0.07 \mathrm{f}$ & $16 \pm 0.06 \mathrm{~g}$ & $11.09 \pm 0.09 \mathrm{~h}$ \\
$\mathrm{GG}$ & & $10.41 \pm 0.02 \mathrm{e}$ & $16.33 \pm 0.05 \mathrm{f}$ & $11.2 \pm 0.13 \mathrm{~h}$ \\
$\mathrm{CH}$ & $11.55 \pm 0.04 \mathrm{c}$ & $18.8 \pm 0.03 \mathrm{~d}$ & $18.1 \pm 0.11 \mathrm{c}$ \\
$\mathrm{CH}+\mathrm{GG}$ & & $12.09 \pm 0.04 \mathrm{~b}$ & $19.45 \pm 0.07 \mathrm{~b}$ & $19.11 \pm 0.1 \mathrm{~b}$ \\
$\mathrm{CK}$ & $6.15 \pm 0.04 \mathrm{k}$ & $13.8 \pm 0.08 \mathrm{j}$ & $10.02 \pm 0.02 \mathrm{j}$ \\
$\mathrm{GG}$ & 40 & $6.33 \pm 0.05 \mathrm{j}$ & $14.1 \pm 0.04 \mathrm{i}$ & $10.2 \pm 0.09 \mathrm{i}$ \\
$\mathrm{CH}$ & & $18.7 \pm 0.08 \mathrm{~d}$ & $14.92 \pm 0.06 \mathrm{f}$ \\
$\mathrm{CH}+\mathrm{GG}$ & & $11.09 \pm 0.09 \mathrm{~d}$ & $19.23 \pm 0.05 \mathrm{c}$ & $17.07 \pm 0.08 \mathrm{~d}$ \\
$\mathrm{CK}$ & 60 & $5.5 \pm 0.02 \mathrm{~m}$ & $10.5 \pm 0.031$ & $9.1 \pm 0.041$ \\
$\mathrm{GG}$ & & $5.72 \pm 0.041$ & $11.96 \pm 0.06 \mathrm{k}$ & $9.41 \pm 0.06 \mathrm{k}$ \\
$\mathrm{CH}$ & & $8.99 \pm 0.08 \mathrm{i}$ & $14.9 \pm 0.02 \mathrm{~h}$ & $13.99 \pm 0.07 \mathrm{~g}$ \\
$\mathrm{CH}+\mathrm{GG}$ & & $9.32 \pm 0.07 \mathrm{~g}$ & $17.7 \pm 0.08 \mathrm{e}$ & $16.53 \pm 0.13 \mathrm{e}$ \\
\hline
\end{tabular}

The means showing same letters in each column are not different statistically $(\mathrm{P} \leq 0.05)$

Chitosan 0\%-Gum ghatti 0\% (CK); Chitosan 0\%-Gum ghatti 1\% (GG); Chitosan 1\%-Gum ghatti 0\% (CH); Chitosan $1 \%$-Gum ghatti $1 \%(\mathrm{CH}+\mathrm{GG})$

\section{Polyamines}

The polyamines such as putrescine, spermidine and spermine at the first time were $12.18,21.35$ and $20.41 \mathrm{nmol} \mathrm{g}^{-1}$ FW of berry for all grapes. As shown in Table 4, there is a dramatic decrease in polyamines such as putrescine, spermidine and spermine in uncoated and GG coated samples compared with other coated samples especially $\mathrm{CH}+\mathrm{GG}$. During 60 days of storage, the highest putrescine, spermidine and spermine was achieved with $\mathrm{CH}+\mathrm{GG}(9.32,17.7$, $16.53 \mathrm{nmol} \mathrm{g}^{-1} \mathrm{FW}$ of berry respectively) and the lowest levels were found in CK sample $\left(5.5,10.5,9.1 \mathrm{nmol} \mathrm{g}^{-1} \mathrm{FW}\right.$ of berry respectively).

\section{Soluble sugars}

The soluble sugars stored in grapes can be in hexoses form (glucose and fructose) dominated with trace sucrose. In our study, a variety of grapes (Rishbaba) showed that glucose content was almost always higher than that of fructose. The effect of $\mathrm{CH}$ and $\mathrm{CH}+\mathrm{GG}$ and their interaction on berry soluble sugar concentration were significant. During 60 days, the greatest fructose concentration was obtained with $\mathrm{CH}$ coated without and/or with $\mathrm{GG}(\mathrm{CH}+\mathrm{GG})$. Moreover, the highest glucose concentration was found in $\mathrm{CH}+$ GG treatment (Table 5). The highest concentrations of soluble sugars were in grapes coated with $1 \%$ of $\mathrm{CH}$ and $1 \%$ GG treatment. The glucose, fructose and sucrose concentrations were the lowest in uncoated grapes (CK).

\section{Mineral contents}

The mineral compositions of grapes are summarized in Table 6. In terms of the content of potassium during the 20th and 40th days, there was a significant difference between CK and GG compared with $\mathrm{CH}+\mathrm{GG}$. As to calcium content, there were significant differences between coated samples and uncoated samples. During 20, 40 and 60 days of storage, there were significant differences between CK and GG samples in comparison with $\mathrm{CH}+\mathrm{GG}$. During 20 days, the effect of all treatments especially $\mathrm{CH}+\mathrm{GG}$ on berry manganese content was significant compared to the controls. During the 60th day, the manganese content was better preserved in $\mathrm{CH}+\mathrm{GG}$ treatment. There were significant differences in the iron content between the control and all treatments samples. There was also a significant difference between the sample treated with $\mathrm{CH}$ alone and GG alone compared with $\mathrm{CH}+\mathrm{GG}$. There was no significant difference in terms of magnesium and zinc content between coated and uncoated samples.

\section{Yeast and mold counts}

The changes in the population of yeast and molds in coated and uncoated grapes are presented in Fig. 3. On the shelf, within the storage times, decay increased. At the end of storage, the decay rate of control $\left(7.3 \log \mathrm{CFU} \mathrm{g}{ }^{-1}\right)$ was significantly higher than other groups $\left(\mathrm{GG}, 7 \log \mathrm{CFU} \mathrm{g}{ }^{-1}\right.$; 
Table 5 The effect of pretreatment with chitosan and gum ghatti on soluble sugars of 'Rishbaba' grape stored at 0 $\pm 1{ }^{\circ} \mathrm{C}$

\begin{tabular}{lcclc}
\hline Treatments & Day & Glucose $(\mu \mathrm{mol} / \mathrm{g} \mathrm{FW})$ & Fructose $(\mu \mathrm{mol} / \mathrm{g} \mathrm{FW})$ & Sucrose $(\mu \mathrm{mol} / \mathrm{g} \mathrm{FW})$ \\
\hline $\mathrm{CK}$ & 0 & $30 \pm 0.1 \mathrm{j}$ & $22.1 \pm 0.1 \mathrm{k}$ & $0.85 \pm 0.01 \mathrm{~d}$ \\
$\mathrm{GG}$ & $30 \pm 0.1 \mathrm{j}$ & $22.1 \pm 0.1 \mathrm{k}$ & $0.85 \pm 0.01 \mathrm{~d}$ \\
$\mathrm{CH}$ & $30 \pm 0.1 \mathrm{j}$ & $22.1 \pm 0.1 \mathrm{k}$ & $0.85 \pm 0.01 \mathrm{~d}$ \\
$\mathrm{CH}+\mathrm{GG}$ & & $30 \pm 0.1 \mathrm{j}$ & $22.1 \pm 0.1 \mathrm{k}$ & $0.85 \pm 0.01 \mathrm{~d}$ \\
$\mathrm{CK}$ & 20 & $30.1 \pm 0.07 \mathrm{j}$ & $22.3 \pm 0.15 \mathrm{k}$ & $0.85 \pm 0.03 \mathrm{~d}$ \\
$\mathrm{GG}$ & & $30.4 \pm 0.06 \mathrm{i}$ & $22.7 \pm 0.13 \mathrm{j}$ & $0.86 \pm 0.02 \mathrm{~d}$ \\
$\mathrm{CH}$ & & $35.1 \pm 0.15 \mathrm{e}$ & $28.1 \pm 0.12 \mathrm{e}$ & $0.94 \pm 0.07 \mathrm{~d}$ \\
$\mathrm{CH}+\mathrm{GG}$ & & $40.6 \pm 0.12 \mathrm{~b}$ & $30.1 \pm 0.1 \mathrm{c}$ & $0.98 \pm 0.03 \mathrm{~d}$ \\
$\mathrm{CK}$ & 40 & $33.1 \pm 0.16 \mathrm{~h}$ & $23.1 \pm 0.2 \mathrm{i}$ & $0.9 \pm 0.01 \mathrm{~d}$ \\
$\mathrm{GG}$ & & $34.5 \pm 0.12 \mathrm{f}$ & $24.1 \pm 0.17 \mathrm{~h}$ & $0.95 \pm 0.04 \mathrm{~d}$ \\
$\mathrm{CH}$ & & $38.5 \pm 0.08 \mathrm{c}$ & $29.9 \pm 0.14 \mathrm{~d}$ & $1.15 \pm 0.02 \mathrm{~d}$ \\
$\mathrm{CH}+\mathrm{GG}$ & & $41.4 \pm 0.06 \mathrm{a}$ & $32.1 \pm 0.11 \mathrm{~b}$ & $1.24 \pm 0.02 \mathrm{c}$ \\
$\mathrm{CK}$ & 60 & $33.6 \pm 0.1 \mathrm{~g}$ & $25.2 \pm 0.26 \mathrm{~g}$ & $1.02 \pm 0.06 \mathrm{~d}$ \\
$\mathrm{GG}$ & & $35.8 \pm 0.15 \mathrm{~d}$ & $26.4 \pm 0.23 \mathrm{f}$ & $1.11 \pm 0.03 \mathrm{~d}$ \\
$\mathrm{CH}$ & & $41.1 \pm 0.1 \mathrm{a}$ & $30.3 \pm 0.2 \mathrm{c}$ & $1.37 \pm 0.05 \mathrm{~b}$ \\
$\mathrm{CH}+\mathrm{GG}$ & & $41.6 \pm 0.15 \mathrm{a}$ & $32.6 \pm 0.15 \mathrm{a}$ & $1.42 \pm 0.02 \mathrm{a}$ \\
\hline
\end{tabular}

The means showing same letters in each column are not different statistically $(\mathrm{P} \leq 0.05)$

Chitosan 0\%-Gum ghatti 0\% (CK); Chitosan 0\%-Gum ghatti 1\% (GG); Chitosan 1\%-Gum ghatti 0\% (CH); Chitosan 1\%-Gum ghatti $1 \%(\mathrm{CH}+\mathrm{GG})$

Table 6 The effect of pretreatment with chitosan and gum ghatti on mineral contents of 'Rishbaba' grape stored at $0 \pm 1{ }^{\circ} \mathrm{C}$

\begin{tabular}{lcrrrrrr}
\hline Treatments & Day & Potassium $(\%)$ & \multicolumn{1}{c}{ Calcium $(\%)$} & Magnesium $(\%)$ & Iron $(\mathrm{ppm})$ & Zinc $(\mathrm{ppm})$ & Manganese $(\mathrm{ppm})$ \\
\hline $\mathrm{CK}$ & 0 & $2 \pm 0.08 \mathrm{a}$ & $0.212 \pm 0.002 \mathrm{a}$ & $0.175 \pm 0.003 \mathrm{a}$ & $25.54 \pm 0.1 \mathrm{a}$ & $13.5 \pm 0.09 \mathrm{a}$ & $9.95 \pm 0.05 \mathrm{a}$ \\
$\mathrm{GG}$ & & $2 \pm 0.08 \mathrm{a}$ & $0.212 \pm 0.002 \mathrm{a}$ & $0.175 \pm 0.003 \mathrm{a}$ & $25.54 \pm 0.1 \mathrm{a}$ & $13.5 \pm 0.09 \mathrm{a}$ & $9.95 \pm 0.05 \mathrm{a}$ \\
$\mathrm{CH}$ & & $2 \pm 0.08 \mathrm{a}$ & $0.212 \pm 0.002 \mathrm{a}$ & $0.175 \pm 0.003 \mathrm{a}$ & $25.54 \pm 0.1 \mathrm{a}$ & $13.5 \pm 0.09 \mathrm{a}$ & $9.95 \pm 0.05 \mathrm{a}$ \\
$\mathrm{CH}+\mathrm{GG}$ & & $2 \pm 0.08 \mathrm{a}$ & $0.212 \pm 0.002 \mathrm{a}$ & $0.175 \pm 0.003 \mathrm{a}$ & $25.54 \pm 0.1 \mathrm{a}$ & $13.5 \pm 0.09 \mathrm{a}$ & $9.95 \pm 0.05 \mathrm{a}$ \\
$\mathrm{CK}$ & 20 & $1.41 \pm 0.05 \mathrm{~b}$ & $0.163 \pm 0.004 \mathrm{f}$ & $0.156 \pm 0.002 \mathrm{c}$ & $18.5 \pm 0.11 \mathrm{e}$ & $12.7 \pm 0.01 \mathrm{~d}$ & $9.09 \pm 0.04 \mathrm{e}$ \\
$\mathrm{GG}$ & & $1.5 \pm 0.09 \mathrm{~b}$ & $0.17 \pm 0.001 \mathrm{f}$ & $0.156 \pm 0.002 \mathrm{c}$ & $20.01 \pm 0.11 \mathrm{~d}$ & $12.75 \pm 0.05 \mathrm{~d}$ & $9.41 \pm 0.03 \mathrm{~d}$ \\
$\mathrm{CH}$ & & $1.72 \pm 0.08 \mathrm{~b}$ & $0.206 \pm 0.002 \mathrm{~b}$ & $0.169 \pm 0.002 \mathrm{~b}$ & $23.17 \pm 0.1 \mathrm{c}$ & $12.92 \pm 0.01 \mathrm{c}$ & $9.7 \pm 0.01 \mathrm{c}$ \\
$\mathrm{CH}+\mathrm{GG}$ & & $1.81 \pm 0.04 \mathrm{~b}$ & $0.211 \pm 0.001 \mathrm{a}$ & $0.174 \pm 0.002 \mathrm{a}$ & $24.12 \pm 0.11 \mathrm{~b}$ & $13.01 \pm 0.05 \mathrm{~b}$ & $9.8 \pm 0.06 \mathrm{~b}$ \\
$\mathrm{CK}$ & 40 & $1.15 \pm 0.02 \mathrm{c}$ & $0.148 \pm 0.003 \mathrm{~g}$ & $0.154 \pm 0.002 \mathrm{c}$ & $16.84 \pm 0.09 \mathrm{~g}$ & $9.65 \pm 0.05 \mathrm{~h}$ & $6.45 \pm 0.01 \mathrm{i}$ \\
$\mathrm{GG}$ & & $1.2 \pm 0.07 \mathrm{c}$ & $0.17 \pm 0.003 \mathrm{f}$ & $0.155 \pm 0.002 \mathrm{c}$ & $18.81 \pm 0.08 \mathrm{~d}$ & $9.76 \pm 0.01 \mathrm{~g}$ & $6.59 \pm 0.05 \mathrm{~h}$ \\
$\mathrm{CH}$ & & $1.55 \pm 0.02 \mathrm{~b}$ & $0.202 \pm 0.001 \mathrm{c}$ & $0.166 \pm 0.004 \mathrm{~b}$ & $19.96 \pm 0.1 \mathrm{~d}$ & $10.22 \pm 0.02 \mathrm{f}$ & $7.01 \pm 0.03 \mathrm{~g}$ \\
$\mathrm{CH}+\mathrm{GG}$ & & $1.61 \pm 0.05 \mathrm{~b}$ & $0.208 \pm 0.001 \mathrm{~b}$ & $0.169 \pm 0.001 \mathrm{~b}$ & $20.01 \pm 0.12 \mathrm{~d}$ & $10.3 \pm 0.01 \mathrm{e}$ & $7.56 \pm 0.01 \mathrm{f}$ \\
$\mathrm{CK}$ & 60 & $1.03 \pm 0.04 \mathrm{~d}$ & $0.133 \pm 0.01 \mathrm{i}$ & $0.146 \pm 0.001 \mathrm{~d}$ & $16.53 \pm 0.15 \mathrm{~h}$ & $6.1 \pm 0.021$ & $4.28 \pm 0.02 \mathrm{~m}$ \\
$\mathrm{GG}$ & & $1.09 \pm 0.01 \mathrm{~d}$ & $0.164 \pm 0.006 \mathrm{f}$ & $0.151 \pm 0.003 \mathrm{c}$ & $17.54 \pm 0.17 \mathrm{f}$ & $6.35 \pm 0.05 \mathrm{k}$ & $4.45 \pm 0.011$ \\
$\mathrm{CH}$ & & $1.22 \pm 0.01 \mathrm{c}$ & $0.19 \pm 0.001 \mathrm{e}$ & $0.163 \pm 0.001 \mathrm{~b}$ & $18.98 \pm 0.0 \mathrm{~d}$ & $7.02 \pm 0.05 \mathrm{j}$ & $5.03 \pm 0.01 \mathrm{k}$ \\
$\mathrm{CH}+\mathrm{GG}$ & & $1.25 \pm 0.01 \mathrm{c}$ & $0.195 \pm 0.003 \mathrm{~d}$ & $0.166 \pm 0.002 \mathrm{~b}$ & $20.05 \pm 0.09 \mathrm{~d}$ & $7.1 \pm 0.02 \mathrm{i}$ & $6 \pm 0.08 \mathrm{j}$ \\
\hline
\end{tabular}

The means showing same letters in each column are not different statistically $(\mathrm{P} \leq 0.05)$

Chitosan 0\%-Gum ghatti 0\% (CK); Chitosan 0\%-Gum ghatti 1\% (GG); Chitosan 1\%-Gum ghatti 0\% (CH); Chitosan 1\%-Gum ghatti 1\% (CH + GG)

$\mathrm{CH}, 6.3 \log \mathrm{CFU} \mathrm{g}{ }^{-1}$ and $\left.\mathrm{CH}+\mathrm{GG}, 6 \log \mathrm{CFU} \mathrm{g}^{-1}\right)$. On the $40^{\text {th }}$ day of storage, the levels of decay for all samples increased clearly. $\mathrm{CH}+\mathrm{GG}$ had the best result on prohibiting the grape decay.

\section{Discussion}

Grape is among the main sources of phenolic compounds, which have good effects on human health by neutralizing 


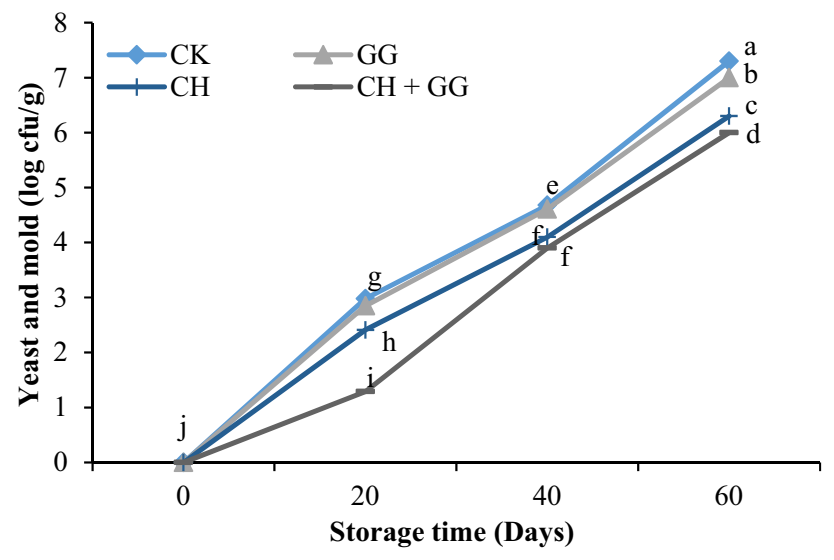

Fig. 3 Effect of chitosan, gum ghatti, chitosan-gum ghatti coatings on yeast and mold counts of grapes stored at $0 \pm 1{ }^{\circ} \mathrm{C}$ with $85 \%$ relative humidity for 60 days. The means showing same letters in each point are not different statistically (P $\leq 0.05$ ). Chitosan $0 \%$-Gum ghatti $0 \%$ (CK); Chitosan 0\%-Gum ghatti 1\% (GG); Chitosan 1\%-Gum ghatti $0 \%(\mathrm{CH})$; Chitosan 1\%-Gum ghatti $1 \%(\mathrm{CH}+\mathrm{GG})$

free radicals. Phenolic acids (Table 1) benefit human health by conferring the ability to sequester reactive oxygen species (ROS) so they have protective effect against chronic diseases [25]. One of the most important extracted from phenolic compounds is resveratrol, as a stilbene. Stilbenes, especially resveratrol, have been warranted that anti-cancer and COVID-19 [26]. Therefore, they protect these components by using $\mathrm{CH}$ and/or GG coatings especially with formula $\mathrm{CH}+\mathrm{GG}$. In addition to its nutraceuticals value, higher preserved resveratrol in grape samples treated with $\mathrm{CH}+\mathrm{GG}$ could protect cells against Botrytis cinerea penetration [27]. The total phenolic and flavonoid are shown in Table 1 and 2 respectively. Phenolic and flavonoid compounds are secondary metabolites of plants, which possess different activities such as antiinflammatory and anticancer effects. It has been established that they can scavenge free radicals produced in the body. Some of these compounds such as gallic acid, caffeic acid, resveratrol, quercetin, etc. are widely used in pharmaceuticals so it is important to preserve them during storage time. Studies have shown that phenol content of the grapes had a great difference due to pre or postharvest treatments, ecological conditions, and genotypic variation [28]. Given the importance of functional foods as potentially healthful products, protecting the phenolic acids and flavonoids of grapes using $\mathrm{CH}$ and $\mathrm{GG}$ (especially $\mathrm{CH}+$ $\mathrm{GG})$ coatings is another major benefit of the coating. The flavonoids such as flavanols and flavan-3-ols have antioxidant activity in the body. The most important compounds of flavan-3-ols (are derivatives of flavans) and flavanols are catechin and quercetin, as main sources in the diet [29]. A sharp decrease of phenolic and flavonoids contents in uncoated grapes may be cell structure breakdown or due to the activity of yeast and mold [30]. Our study suggests that coating with $\mathrm{CH}$ based formula without and/or with $\mathrm{GG}$, especially $\mathrm{CH}+\mathrm{GG}$, is a suitable method for preventing activity of yeast and mold during storage period. These observations are consistent with other studies. For example, arabic gum lowered the reduction of phenolic contents in banana [31]. In addition, in strawberry fruit, Aloe vera gel coating enriched with ascorbic acid, calcium lactate and cinnamon essential oil protected phenolic compound [32]. Since the phenolic acids and flavonoids are the most important group of natural polyphenols and have a protective role against oxidations, so protecting them by using $\mathrm{CH}$ and $\mathrm{GG}$ (especially $\mathrm{CH}+\mathrm{GG}$ ) coatings is another major benefit of the coating. Indeed, the higher stability of phenolic compound in samples treated with $\mathrm{CH}+\mathrm{GG}$ confirms the positive effect of this polysaccharides ability for preserving both nutritional and antioxidant of treated berry during cold storage [27].

Grape by-products are promising and sustainable sources of anthocyanidins (Table 3). Anthocyanidins stability was influenced by the presence of hydroxyl or methoxyl groups and B-ring in the structure. Anthocyanidins, as natural colorants, have value-added properties [33]. $\mathrm{CH}$ and GG coatings protected grape anthocyanidin compounds. In this research, grapes coated with high concentrations of $\mathrm{CH}+\mathrm{GG}$ showed significantly less reduction of anthocyanidins than the other treatments. These compounds are highly nutrient and antioxidant with many other good effects on health. There was a relationship between polyamines, total phenol contents and anthocyanins changes. Reduction of anthocyanin may be because of coupled oxidation processes in the presence of other phenolics [27, 34]. $\mathrm{CH}+\mathrm{GG}$ treatment reduced water loss, restricted gas exchange, and delayed ripening. According to the results of this study, coating with $\mathrm{CH}$-based formula without and/or with GG, especially $\mathrm{CH}+\mathrm{GG}$ markedly delayed the decrease of anthocyanin contents (Table 3). Wang and Gao studied strawberry coated with 0.5, 1.0 and $1.5 \mathrm{~g} 100 \mathrm{~mL}^{-1} \mathrm{CH}$ solutions kept at 5 or $10^{\circ} \mathrm{C}$. They maintained that anthocyanin was greater in the coated samples compared to the uncoated samples [35]. Their finding is in agreement with our results. Because grapes are widely consumed in the world so the evaluation of its bioactive compounds such as antioxidant capacity (DPPH assay) is important. The antioxidant capacity decreased during the storage time. $\mathrm{CH}+\mathrm{GG}$ coating are considered an effective strategy to preserve these compounds (Fig. 1). As known, some compounds like phenolics, flavonoids, and anthocyanins support antioxidant activity in grape and other fruits [27]. Antioxidant capacity and total phenol showed that phenolic alone made no notable contribution to antioxidant activity. Antioxidant capacity may depend on phenols and phenolic acids, flavonoids, lignans, tannins, coumarins, and quinones 
stilbenes rather than total phenol content. Sanchez-Gonzalez et al. studied Muscatel table grapes coated with $\mathrm{CH}(1 \%)$, hydroxypropylmethylcellulose (1\%) and bergamot essential oil (2\%). They found no significant difference between the coated and control samples in terms of total phenol content [36]. These results were almost similar to the results of our research, although in our study, the combination of $\mathrm{CH}+$ GG has caused significant changes in reducing these parameters and compounds such as polyphenols, flavonoids were examined in detail.

$\mathrm{CH}+\mathrm{GG}$ can control defense-related enzymes activity and improve fruits preservation. Polyphenol oxidase is responsible for fruit browning. The activity of this enzyme depends on the grading of the fruit, temperatures, $\mathrm{pH}$ and various other conditions during storage [37, 38]. In addition, the lower PPO activity can be because of the antioxidant activity of $\mathrm{CH}+\mathrm{GG}$ coating carrier or $\mathrm{CH}+\mathrm{GG}$ inhibits polyphenol oxidase by reacting with the active site of the enzyme (Fig. 2). The inhibitory effect on PPO activity in the treated samples can be because of the GG incorporated into $\mathrm{CH}$-based coating.

Polyamines are correlated with numerous plant processes. They effectively maintain berry firmness and stabilize anthocyanins along with suppressing the activity of pectin methylesterase [39]. Coating with $\mathrm{CH}+\mathrm{GG}$ protected the grape polyamines (Table 4), which are important players in decreasing stress and disease resistance, such as Alzheimer's or infectious diseases.

The effect of $\mathrm{CH}, \mathrm{CH}+\mathrm{GG}$ and their interaction on grape soluble sugar concentration are listed in Table 5. In this study, soluble sugars increased and a significant difference was observed between the control sample and the treatments, which could be due to polyphenol oxidase activity and acidity changes. On the other hand, changes in soluble sugars due to cellular respiration and conversion of disaccharides to monosaccharides have been increasing. Since the main factors in grape sensory characteristics and quality are the soluble sugars fructose, glucose, and sucrose [40], the effects of all treatments on these factors were examined that $\mathrm{CH}+\mathrm{GG}$ treatment plays a major role in protecting the soluble sugars.

Grape is a good source of minerals such as copper, manganese, potassium, iron, phosphorus, magnesium and zinc only in trace amounts [41]. Minerals are as effective as phenolic contents in terms of health benefits. Studies have addressed the benefits of metals in biological systems [42]. Potassium helps the growth, repair and maintenance of bones and along with sodium, it keeps the balance of electrochemical charges in cellular tissue. Calcium was one of the best-retained nutrients in the fresh grapes evaluated throughout this study [41]. Manganese improves bone development and hormone synthesis. It is part of the structure of the superoxide dismutase enzyme and supports the function of the nervous system. Iron is needed to synthesize red blood cells and adenosine triphosphate or ATP. Magnesium is important in the synthesis of DNA and RNA, the formation and function of ATP and many other enzymatic reactions [41]. Zinc is one of the main trace minerals so that the body only needs a small amount it therefore, it is better to prevent the reduction of these essential substances in the fruit by coating the grape fruits. Coated with $\mathrm{CH}$-based formula without and/or with GG, especially $\mathrm{CH}+\mathrm{GG}$ markedly delayed the changes because it has been able to control enzymatic activity by reducing respiration. The results for each mineral were different, still all of them decreased especially in uncoated samples (Table 6).

In our research, the results showed that the $\mathrm{CH}$ coatings decreased the decay of grapes (Fig. 3). Two mechanisms were explained about $\mathrm{CH}$ effect on decreasing the growth of yeast and mold; (1) $\mathrm{CH}$ leads to the loss of proteinaceous constituents and intracellular electrolytes, (2) affecting mRNA and protein synthesis. The mechanism of microorganism inhibition by $\mathrm{CH}+\mathrm{GG}$ is that coatings obtained by hydrophilic polymers, like guar gum, GG, and potato starch exhibited gas permeability that decreases with a decrease in temperature and relative humidity [43, 44]. On the other hand, phenolic compounds such as resveratrol, which are stabilized in the grape fruit by $\mathrm{CH}+\mathrm{GG}$, have antimicrobial properties and delay the growth of mold and yeast. As far as the authors know, the present study is the first work to demonstrate that using a mixture of $\mathrm{CH}$ and $\mathrm{GG}$ at different sub-inhibitory concentrations can significantly inhibit the yeast and mold on table grapes stored for 60 days.

\section{Conclusion}

The results of our study showed that coatings of grape using $\mathrm{CH}$-based formula with and without GG preserved nutrients and maintain quality within 60 days at $0 \pm 1{ }^{\circ} \mathrm{C}$ and $85 \%$ relative humidity. Coatings especially $\mathrm{CH}+\mathrm{GG}$ inhibited decay incidence and delayed changes in the contents of nutritional properties, phenolic compounds and antioxidant capacity. The coatings showed positive effects on soluble sugars and inhibited the PPO activities. Moreover, an investigation of bacterial activity showed that treatments with chitosan coating and gum ghatti provided a significant reduction in yeast-mold growth. In comparison to other combined coatings, $\mathrm{CH}$ and GG, as a new edible coating, are considered biodegradable and less hazardous with a significant role in decreasing postharvest losses of grape fruits. The $\mathrm{CH}+$ GG treatment had the highest effect on many components of grape fruits so studying this coating showed an investigate increase of the chitosan-gumghatti coating applications to diminish the postharvest damage by microorganisms, as well as the improvement of the quality of the other fruits. 
Funding Funding was provided by Malayer University of Iran (R. Karimi; Grant no. 84.5-310). The authors gratefully acknowledge the Malayer University.

Data availability The authors declare that data supporting the findings of this study are available within the article.

\section{References}

1. R. Karimi, Cold hardiness evaluation of 20 commercial table grape (Vitis vinifera L.) cultivars. Int. J. Fruit Sci. 20(3), 433-450 (2020)

2. V. Kedage, J. Tilak, G. Dixit, T. Devasagayam, M. Mhatre, A study of antioxidant properties of some varieties of grapes (Vitis vinifera L.). Crit. Rev. Food Sci. Nutr. 47, 175-185 (2007)

3. E. Feliziani, J.L. Smilanick, D.A. Margosan, M.F. Mansour, G. Romanazzi, S. Gu, H.L. Gohil, Z.R. Ames, Preharvest fungicide, potassium sorbate, or chitosan use on quality and storage decay of table grapes. Plant Dis. 97(3), 307-314 (2013)

4. X. Zhou, J. Tan, Y. Gou, Y. Liao, F. Xu, G. Li, J. Cao, J. Yao, J. Ye, N. Tang, Z. Chen, The biocontrol of postharvest decay of table grape by the application of kombucha during cold storage. Sci Hortic. 253, 134-139 (2019)

5. K. Wang, Y. Liao, S. Cao, H. Di, Y. Zheng, Effects of benzothiadiazole on disease resistance and soluble sugar accumulation in grape berries and its possible cellular mechanisms involved. Postharvest Biol Technol. 102 (2015)

6. A. Milani, M. Jouki, M. Rabbani, Production and characterization of freeze-dried banana slices pretreated with ascorbic acid and quince seed mucilage: physical and functional properties. Food Sci Nutr. 8, 3768-3776 (2020)

7. F. Alipoorfard, M. Jouki, H. Tavakolipour, Application of sodium chloride and quince seed gum pretreatments to prevent enzymatic browning, loss of texture and antioxidant activity of freeze dried pear slices. J Food Sci Technol. 57, 3165-3175 (2020)

8. S. Paidari, N. Zamindar, R. Tahergorabi, M. Kargar, Sh. Ezzat, N. Shirani, H. Musavi, Edible coating and films as promising packaging: a mini review. Food Measure. Charact. 15, 4205-4214 (2021)

9. N.M. Asl, H. Ahari, A.A.M. Moghanjoghi, S. Paidari, Assessment of nanochitosan packaging containing silver NPs on improving the shelf life of caviar (Acipenser persicus) and evaluation of nanoparticles migration Food Measure. Character. 15, 5078-5086 (2021)

10. A. Ashrafi, M. Jokar, A. Mohammadi Nafchi, Preparation and characterization of biocomposite film based on chitosan and kombucha tea as active food packaging. Int. J. Biol. Macromol. 108, 444-454 (2018)

11. N. Oladzadabbasabadi, A. Mohammadi Nafchi, F. Ariffin, M.M.J.O. Wijekoon, A.A. Al-Hassan, M.A. Dheyab, M. Ghasemlou, Recent advances in extraction, modification, and application of chitosan in packaging industry. Carbohydr Polym 277, 118876 (2022)

12. A. Joshi, N.S. Baraiya, P.B. Vyas, T.V.R. Rao, Gum ghatti based edible coating emulsion with an additive of clove oil improves the storage life and maintains the quality of Papaya (Carica papaya L., cv Madhu bindu). Int J Curr Microbiol Appl Sci. 6, 160-174 (2017)

13. S. Eshghi, R. Karimi, A. Shiri, M. Karami, M. Moradi, The novel edible coating based on chitosan and gum ghatti to improve the quality and safety of "Rishbaba" table grape during cold storage. Food Measure. 15, 3683-3693 (2021)
14. Y. Jiang, Y. Li, Effects of chitosan coating on postharvest life and quality of longan fruit. Food Chem. 73(2), 139-143 (2001)

15. J. Koponen, A. Happonen, P. Mattila, R. Torronen, Contents of anthocyanins and ellagitannins in selected foods consumed in Finland. J Agric Food Chem. 55, 1612-1619 (2007)

16. V.L. Singleton, J.A. Rossi, Colorimetry of total phenolics with phosphomolybdic phosphotungstic acid reagents. Am. J. Enol. Vitic. 16(3), 144-158 (1965)

17. J. Zhishen, T. Mengcheng, W. Jianming, The determination of flavonoid contents in mulberry and their scavenging effects on superoxide radicals. Food Chem. 64(4), 555-559 (1999)

18. M. Downey, M. Mazza, M. Krstic, Development of a stable extract for anthocyanins and flavonols from grape skin. Am. J. Enol. Vitic. 58, 358-364 (2007)

19. D.T. Krizek, S.J. Britz, R.M. Mirecki, Inhibitory effects of ambient levels of solar UV-A and UV-B radiation on growth of cv. New Red Fire lettuce. Physiol. Plant. 103(1), 1-7 (1998)

20. M. Jouki, N. Khazaei, S. Rashidi-Alavijeh, S. Ahmadi, Encapsulation of Lactobacillus casei in quince seed gum-alginate beads to produce a functional synbiotic drink powder by agro-industrial byproducts and freeze-drying. Food Hydrocoll. 120, 106895 (2021)

21. S. Eshghi, M. Hashemi, A. Mohammady, F. Badii, Z. Mohammadhoseini, K. Ahmadi, Effect of nanochitosan-based coating with and without copper loaded on physicochemical and bioactive components of fresh Strawberry fruit (Fragaria x ananassa Duchesne) during storage. Food Bioprocess Technol. 7, 2397-2409 (2014)

22. H. Walter, J. Geuns, High speed hplc analysis of polyamines in plant tissues. Plant physiol. 83, 232-234 (1987)

23. R. Karimi, M. Koulivand, N. Ollat, Soluble sugars, phenolic acids and antioxidant capacity of grape berries as affected by iron and nitrogen. Acta Physiol. Plant. 41(7), 117 (2019)

24. S. Jafari, M. Jouki, M. Soltani, Modification of physicochemical, structural, rheological, and organoleptic properties of sweetened condensed milk by maltodextrin, fructose, and lactose. Food Measure. Character. 15, 3800-3810 (2021)

25. F. Cosme, T. Pinto, A. Vilela, Phenolic compounds and antioxidant activity in grape juices: a chemical and sensory view. Beverages. 4(1), 22 (2018)

26. Z.S. Asgarian, R. Karimi, M. Ghabooli, M. Maleki, Biochemical changes and quality characterization of cold-stored 'Sahebi' grape in response to postharvest application of GABA. Food Chem. 373, 131401 (2021)

27. H.M. Wahedi, S. Ahmad, S.W. Abbasi, Stilbene-based natural compounds as promising drug candidates against COVID-19. J Biomol Struct Dyn. 39(9), 3225-3234 (2020)

28. F. Sabir, A. Sabir, Postharvest quality maintenance of table grapes cv. 'alphonse lavallée' by exogenous applications of salicylic acid, oxalic acid and MAP. Erwerbs-obstbau (2017). https://doi.org/10. 1007/s10341-016-0314-6

29. A.N. Panche, A.D. Diwan, S.R. Chandra, Flavonoids: an overview. J. Nutr. Sci. 5, e47 (2016)

30. G. Khaliq, A. Saleh, G.A. Bugti, K.R. Hakeem, Guggul gum incorporated with basil essential oil improves quality and modulates cell wall-degrading enzymes of jamun fruit during storage. Sci. Hortic. 273, 109608 (2020)

31. A.A. Alali, M.A. Awad, A.D. Al-Qurashi, S.A. Mohamed, Postharvest gum Arabic and salicylic acid dipping affect quality and biochemical changes of 'Grand Nain' bananas during shelf life. Sci. Hortic. 237, 51-58 (2018)

32. Y. Esmaeili, N. Zamindar, S. Paidari, S.A. Ibrahim, A. Mohammadi Nafchi, The synergistic effects of aloe vera gel and modified atmosphere packaging on the quality of strawberry fruit. J. Food Process. Preserv. 00, e16003 (2021)

33. H.E. Khoo, A. Azlan, S.T. Tang, S.M. Lim, Anthocyanidins and anthocyanins: colored pigments as food, pharmaceutical 
ingredients, and the potential health benefits. Food Nutr Res. 61(1) (2017)

34. X. Pang, H. Xue-mei, Y. Xiao-tang, J.I. Zuo-liang, Z. Zhao-qi, Role of polyphenol oxidase in anthocyanin degradation of Lychee pericarp. Sci. Agric. Sin. 41 (2008)

35. S. Wang, H. Gao, Effect of chitosan-based edible coating on antioxidants antioxidant enzyme system and postharvest fruit quality of strawberries (Fragaria $\times$ aranassa Duch). LWT - Food Sci Technol. 52, 71-79 (2013)

36. L. Sanchez-Gonzalez, C. Pastor, M. Vargas, A. Chiralt, C. Gonzalez-Martinez, M. Chafer, Effect of hydroxypropylmethylcellulose and chitosan coatings with and without bergamot oil on quality and safety of cold-stored grapes. Postharvest Biol. Technol. 60, 57-63 (2011)

37. A. Shekari, R. Naghshiband Hassani, M. Soleimani Aghdam, Exogenous application of GABA retards cap browning in Agaricus bisporus and its possible mechanism. Postharvest Biol Technol. 174, 111434 (2021)

38. G. Adiletta, L. Zampella, C. Coletta, M. Petriccione, Chitosan coating to preserve the qualitative traits and improve antioxidant system in fresh Figs (Ficus carica L.). Agriculture 9, 84 (2019)

39. N. Minois, D. Carmona-Gutierrez, F. Madeo, Polyamines in aging and disease. Aging 3(8), 716-732 (2011)
40. H. Orak, Determination of glucose and fructose contents of some important red grape varieties by HPLC. Asian J. Chem. 21, 3068 3072 (2009)

41. K. Soetan, C. Olaiya, O. Oyewole, The importance of mineral elements for humans, domestic animals and plants: a review. Afr J Food Sci. 4, 200-222 (2009)

42. C. Dani, L.S. Oliboni, D. Prá, D. Bonatto, C.E.I. Santos, M.L. Yoneama, J. Dias, M. Salvador, J. Henriques, Mineral content is related to antioxidant and antimutagenic properties of grape juice. Genet. Mol. Res. 11, 3154-3163 (2012)

43. P.J. Chien, F. Sheu, F.H. Yang, Effects of edible chitosan coating on quality and shelf life of sliced mango fruit. J Food Eng. 78, 225-229 (2007)

44. A. Perdones, L. Sanchez-Gonzalez, A. Chiralt, M. Vargas, Effect of chitosan-lemon essential oil coatings on storage-keeping quality of strawberry. Postharvest Biol. Technol. 70, 32-41 (2012)

Publisher's Note Springer Nature remains neutral with regard to jurisdictional claims in published maps and institutional affiliations. 